Alma Mater Studiorum - Università di Bologna DEPARTMENT OF ECONOMICS

\title{
Strategic Delegation under Cost Asymmetry
}

Flavio Delbono

Luca Lambertini

Luigi Marattin

Quaderni - Working Paper DSE $N^{\circ} 1016$

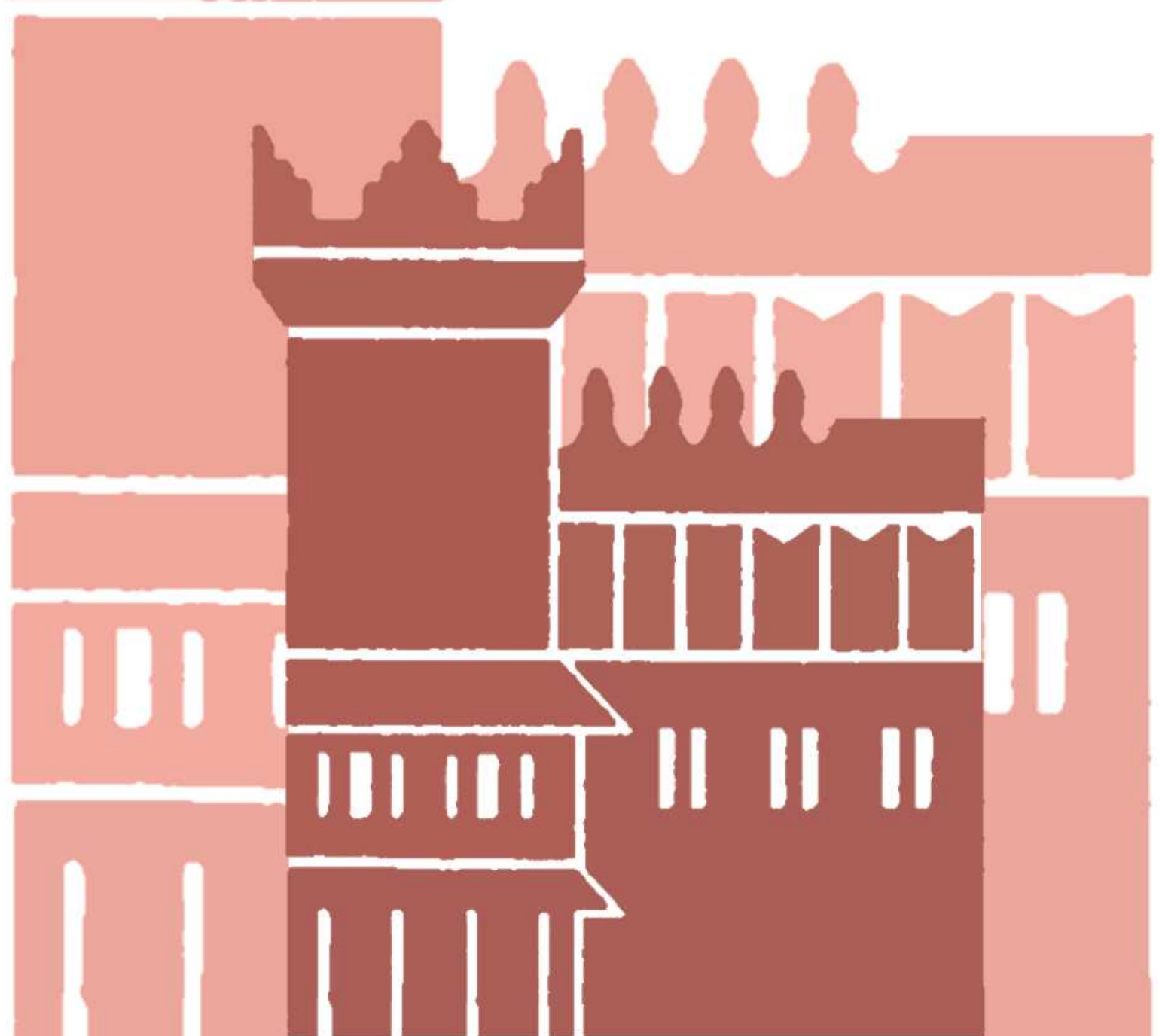




\title{
Strategic Delegation under Cost Asymmetry
}

\author{
Flavio Delbono\#, Luca Lambertini ${ }^{\S}$ and Luigi Marattin ${ }^{\S}$ \\ \# Department of Economics, University of Bologna \\ Piazza Scaravilli 2, 40126 Bologna, Italy \\ flavio.delbono@unibo.it \\ $\S$ Department of Economics, University of Bologna \\ Strada Maggiore 45, 40125 Bologna, Italy \\ luca.lambertini@unibo.it; luigi.marattin@unibo.it
}

July 15, 2015

\begin{abstract}
We revisit the two-stage duopoly game with strategic delegation and asymmetric technologies of Sen and Stamatopoulos (2015). We show that their conclusions are misled by the restrictive assumption that the extent of delegation to managers is restricted to a binary set. Allowing for a continuous set of delegation incentives, we prove that the delegation stage is a prisoners' dilemma, the unique subgame perfect equilibrium entailing both firms hiring managers. At equilibrium, the more efficient firm makes higher profits.
\end{abstract}

JEL Codes: D43, L13

Keywords: managerial firms; Cournot competition; prisoners' dilemma 


\section{Introduction}

In a recent paper, Sen and Stamatopoulos (2015) examine a Cournot duopoly with asymmetric cost functions and strategic delegation. As for the latter, each firm can offer its manager a contract based on either pure profits or pure revenues. They show that there are two pure-strategy equilibria where one firm chooses the revenue contract while the other chooses the profit contract (which is equivalent to not hiring a manager). In one of these equilibria, the inefficient obtains higher profits than the rival.

Here, we reformulate their game by removing their restrictive assumption according to which delegation is chosen from a binary set, allowing owners to choose the extent of delegation as a continuous variable, in accordance with the acquired literature since Vickers (1985). By doing so, we show that (i) the subgame perfect equilibrium is unique; (ii) it involves both firms delegating control to managers over a mix of profits and revenues; (iii) it is the outcome of a prisoners' dilemma; and (iv) the more efficient firm makes higher profits than the rival.

The remainder of the paper is organised as follows. The setup is laid out in section 2 . The subgame perfect equilibrium analysis is in section 3 . Section 4 concludes.

\section{The model}

Setup and notation are the same as in Sen and Stamatopoulos (2015), SS2015 henceforth. Two firms, $A$ and $B$, operate in a market for a homogeneous good whose market demand is $p=\max \{0, k-Q\}$, with $k>0$. Each firm uses a constant returns technology summarised by the cost function $C_{i}=\tau_{i} q_{i}$, where parameter $\tau_{i}>0$ is average and marginal cost. The technological 
asymmetry is captured by the chain of inequalities

$$
k>\tau_{B}>\tau_{A}>0 .
$$

The profit function of firm $i$ is $\pi_{i}=\left(p-\tau_{i}\right) q_{i}$. The game has a twostage structure. In the first stage, owners choose the extent of delegation to managers (if any). In the second, firms (either managerial or entrepreneurial) simultaneously compete in output levels on the market place. If firm $i$ 's owners hire a manager, the latter is offered a contract establishing that the manager has to choose output so as to maximise

$$
\pi_{i}^{\lambda_{i}}\left(q_{A}, q_{B}\right)=p q_{i}-\lambda_{i} \tau_{i} q_{i}
$$

where $\lambda_{i} \geq 0$ is a strategic variable in the owners' hands, to be chosen at the first stage of the game, before market competition takes place. The managerial objective function (2) can be rewritten as $\pi_{i}^{\lambda_{i}}\left(q_{A}, q_{B}\right)=p q_{i}-\lambda_{i} C_{i}$, i.e., as a weighted difference between revenue and cost.

This approach to managerial firms has been pioneered by Vickers (1985), in a pathbreaking paper. There, the managerial firm maximises $M_{i}=\pi_{i}+$ $\theta_{i} q_{i}$, where $\theta_{i} \geq 0$ is the weight given to output, to be set by stockholders at the first stage. Now observe that Vickers's maximand can be rewritten as

$$
M_{i}=p q_{i}-C_{i}+\theta_{i} q_{i}=p q_{i}-\left(\tau_{i}-\theta_{i}\right) q_{i}
$$

It is immediate to detect that $\pi_{i}^{\lambda_{i}}\left(q_{A}, q_{B}\right)=M_{i}$ iff

$$
\lambda_{i}=\frac{\tau_{i}-\theta_{i}}{\tau_{i}}
$$

If so, then the two models, i.e., Vickers (1985) and SS2015, are indeed isomorphic. Moreover, we also know from existing literature that Vickers's model is isomorphic to Fershtman and Judd (1987) and Sklivas (1987), where the managerial maximand is $M_{i}=\alpha_{i} \pi_{i}+\left(1-\alpha_{i}\right) p q_{i}$, i.e., a weighted average 
of profits and revenues (see Lambertini and Trombetta, 2002). All of these structures being isomorphic to each other, they must necessarily generate the same subgame perfect equilibria. In a nutshell, the conclusion emerging from this well established literature since Vickers (1985) is that the extent of delegation in a Cournot setting is strictly positive whenever a manager is higher, and it is determined endogenously at the first stage of the game on the basis of demand and cost parameters. In SS2015, $\lambda_{i} \in\{0,1\}$, i.e., the extent of delegation is chosen from a binary set. In view of the above discussion, this entails that $\lambda_{i}=1$ is indeed admissible because it captures the case in which either the manager is absent or he's given a strict instruction to maximise profits, while the opposite case of revenue maximisation with $\lambda_{i}=0$ is in fact never subgame perfect. This is precisely what we are about to show in the remainder. As a consequence, we shall show that the upstream stage is not an anticoordination game - as claimed by SS2015 - but rather a prisoners' dilemma with a unique equilibrium in dominant strategies where both firms hire managers.

\section{The subgame perfect equilibrium}

The subgame perfect equilibrium obtains by backward induction. Hence, we first have to look at the market stage, where three different situations may arise, whereby we have to characterize three market subgames:

- $(e, e)$ : Both firms are strict profit-seeking units, with $e$ standing for entrepreneurial

- $(m, e)$ or $(e, m)$ : One firm delegates and the other one does not, with $m$ standing for managerial

- $(m, m)$ : Both firms delegate 
In order to ensure the positivity of output levels in all outcomes, we assume that

$$
0<\tau_{A}<\tau_{B}<\widehat{\tau} \equiv \frac{k+7 \tau_{A}}{8}<k,
$$

which differs from the assumption appearing in SS2015 (p. 149, expression (8)). This difference is generated by the fact that here we treat $\lambda_{i}$ as a continuous variable to be chosen endogenously.

The first case entails $\lambda_{A}=\lambda_{B}=1$, as in SS2015. In the resulting asymmetric Cournot equilibrium, profits are:

$$
\pi_{i}(e, e)=\frac{\left(k-2 \tau_{i}+\tau_{j}\right)^{2}}{9}
$$

which is the square of $q_{i}(e, e)$. Given (1), the positivity of firm B's output is ensured by any

$$
\tau_{B} \in\left(\tau_{A}, \frac{k+\tau_{A}}{2}\right)
$$

with $\left(k+\tau_{A}\right) / 2>\widehat{\tau}$. This is the only case coinciding with the analysis in SS2015.

Now we can tackle the asymmetric setup where firm $i$ is managerial while firm $j$ is entrepreneurial (with $\lambda_{j}=1$ ), and $\lambda_{i}$ is chosen endogenously. The profit of firm $i$ is

$$
\pi_{i}(m, e)=\frac{\left[k+\left(\lambda_{i}-3\right) \tau_{i}+\tau_{j}\right]\left(k-2 \lambda_{i} \tau_{i}+\tau_{j}\right)}{9}
$$

and, at the first stage, the owner of firm $i$ must solve:

$$
\frac{\partial \pi_{i}(m, e)}{\partial \lambda_{i}}=-\frac{\tau_{i}\left[k+2\left(2 \lambda_{i}-3\right) \tau_{i}+\tau_{j}\right]}{9}=0
$$

w.r.t. $\lambda_{i}$, whose optimal value is: ${ }^{1}$

$$
\lambda_{i}^{*}(m, e)=\frac{6 \tau_{i}-\tau_{j}-k}{4 \tau_{i}}
$$

\footnotetext{
${ }^{1}$ Second order conditions for profit maximization are satisfied everywhere and therefore omitted for brevity.
} 
The resulting output levels are $q_{i}^{*}(m, e)=\left(k-2 \tau_{i}+\tau_{j}\right) / 2$ and $q_{j}^{*}(e, m)=$ $\left(k+2 \tau_{i}-3 \tau_{j}\right) / 4$, with all quantities being positive, irrespective of which firm is hiring a manager, if $\tau_{B} \in\left(\tau_{A},\left(k+2 \tau_{A}\right) / 3\right)$. Moreover, the efficient firm's output must not exceed the pure monopoly output when the same firm is managerial, i.e., $q_{A}(m, e) \leq\left(k-\tau_{A}\right) / 2$, which holds when (5) is satisfied. The resulting profits are

$$
\begin{aligned}
\pi_{i}^{*}(m, e) & =\frac{\left(k-2 \tau_{i}+\tau_{j}\right)^{2}}{8} \\
\pi_{j}^{*}(e, m) & =\frac{\left(k-3 \tau_{i}+2 \tau_{j}\right)^{2}}{16}
\end{aligned}
$$

The last case is the one where both firms delegate. Here, the relevant first order condition (FOC) for the stockholders of firm $i$ at the upstream stage is:

$$
\frac{\partial \pi_{i}(m, m)}{\partial \lambda_{i}}=-\frac{\tau_{i}\left[k-2 \tau_{i}\left(3-2 \lambda_{i}\right)+\lambda_{j} \tau_{j}\right]}{9}=0
$$

Solving the system (12), we obtain:

$$
\lambda_{i}^{*}(m, m)=-\frac{k-8 \tau_{i}+2 \tau_{j}}{5 \tau_{i}}
$$

generating the following profits:

$$
\pi_{i}^{*}(m, m)=\frac{2\left(k-3 \tau_{i}+2 \tau_{j}\right)^{2}}{25}
$$

Before delving into the details of the first stage of the game, it is worth showing the equivalence between the present model and Vicker's (1985). ${ }^{2}$ For the sake of brevity, we shall confine to the case in which both firms delegate. This, in Vickers's formulation, amounts to saying that managers are given an incentive based on (3). At the market stage, the FOC is:

$$
\frac{\partial M_{i}}{\partial q_{i}}=k-2 q_{i}-q_{j}-\tau_{i}+\theta_{i}=0
$$

\footnotetext{
${ }^{2}$ Here we are reformulating the Vickers (1985) model under asymmetric technologies, which, to the best of our knowledge, has not been done so far.
} 
so that equilibrium output levels are

$$
q_{i}^{V}(m, m)=\frac{k+2 \theta_{i}-\theta_{j}-2 \tau_{i}+\tau_{j}}{3}
$$

where superscript $V$ mnemonics for Vickers. At the delegation stage, firm $i$ 's FOC is:

$$
\frac{\partial \pi_{i}}{\partial q_{i}}=\frac{k-4 \theta_{i}-\theta_{j}-2 \tau_{i}+\tau_{j}}{9}=0
$$

and the optimal extent of delegation is $\theta_{i}^{V}(m, m)=\left(k-3 \tau_{i}+2 \tau_{j}\right) / 5$.

If only firm $i$ delegates control to a manager, expression (16) delivers the asymmetric output levels

$$
q_{i}^{V}(m, e)=\frac{k+2 \theta_{i}-2 \tau_{i}+\tau_{j}}{3} ; q_{j}^{V}(e, m)=\frac{k-\theta_{i}-2 \tau_{i}+\tau_{j}}{3}
$$

and the optimal contract set by the owners of firm $i$ is summarised by $\theta_{i}^{V}(m, e)=\left(k-2 \tau_{i}+\tau_{j}\right) / 4$.

On these bases, one can prove:

Proposition 1 Under constant returns to scale, a delegation contract based on revenues and costs is equivalent to one based on profits and output level.

Proof. This claim can be shown to hold true using (4). When both firms delegate,

$$
\lambda_{i}^{*}(m, m)=\frac{\tau_{i}-\theta_{i}^{V}(m, m)}{\tau_{i}}
$$

Likewise, $\lambda_{i}^{*}(m, e)=\left[\tau_{i}-\theta_{i}^{V}(m, e)\right] / \tau_{i}$ in the asymmetric case in which only firm $i$ delegates. As a consequence all remaining equilibrium magnitudes coincide in SS2015 and Vickers (1985).

The above Proposition indeed proves that the two delegation models are isomorphic, and therefore, by virtue of the analysis in Lambertini and Trombetta (2002), they are also isomorphic to Fershtman and Judd (1987) and Sklivas (1987). Consequently, the subgame perfect equilibrium in our reformulation of SS2015, with the extent of delegation is made endogenous, has 
to coincide with that we already know since Vickers (1985). This is what we are going to illustrate.

The upstream stage in reduced form is depicted by Matrix 1.

$$
\begin{aligned}
& \text { B }
\end{aligned}
$$

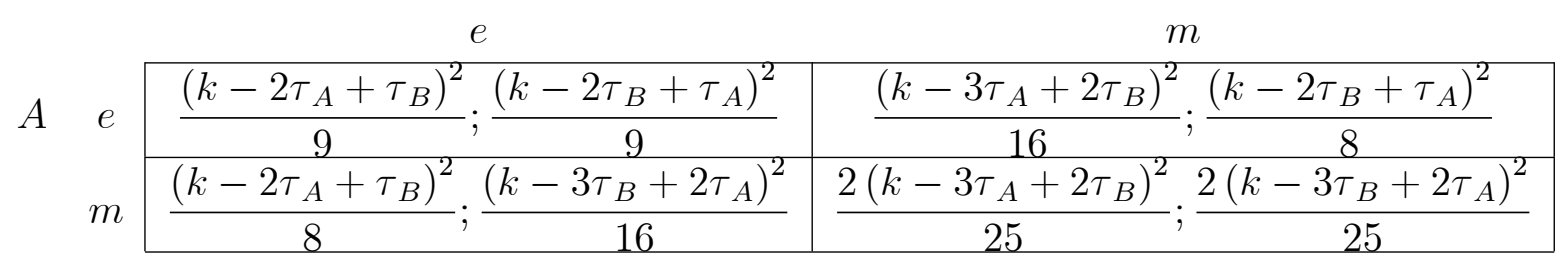

\section{Matrix 1}

Proposition 2 The first stage of the game has a unique Nash equilibrium in pure strategies at $(m, m)$, at the intersection of strictly dominant strategies.

Proof. The above statement results from the following inequalities:

$$
\begin{gathered}
\pi_{i}^{*}(m, e)-\pi_{i}^{*}(e, e)=\frac{\left(k-2 \tau_{i}+\tau_{j}\right)^{2}}{72}>0 \\
\pi_{i}^{*}(m, m)-\pi_{i}^{*}(e, m)=\frac{7\left(k-3 \tau_{i}+2 \tau_{j}\right)^{2}}{400}>0
\end{gathered}
$$

This proves the claim.

In the unique equilibrium $(m, m)$, the profit ranking is determined by the sequence of marginal costs, i.e., $\pi_{A}^{*}(m, m)>\pi_{B}^{*}(m, m)$ because $\tau_{A}<\tau_{B}$. Hence,

Remark 3 At the unique subgame perfect equilibrium, the more efficient firms makes higher profits.

Something more can be told about the nature of the equilibrium generated by the first stage of the game. As we know from Vickers (1985) and the ensuing literature, if firms share the same technology (i.e., $\tau_{A}=\tau_{B}=\tau$ ), 
the game represented in Matrix 1 is a prisoners' dilemma, its equilibrium being inefficient for firms which would be better off by remaining pure profitseeking agents. ${ }^{3}$

In the present setup, marginal costs being asymmetric, we have to evaluate the following expressions:

$$
\begin{aligned}
& \pi_{A}^{*}(e, e)-\pi_{A}^{*}(m, m)=\frac{7 k^{2}+2 k\left(4 \tau_{A}-11 \tau_{B}\right)-62 \tau_{A}^{2}+116 \tau_{A} \tau_{B}-47 \tau_{B}^{2}}{225} \\
& \pi_{B}^{*}(e, e)-\pi_{B}^{*}(m, m)=\frac{7 k^{2}+2 k\left(4 \tau_{B}-11 \tau_{A}\right)-62 \tau_{B}^{2}+116 \tau_{A} \tau_{B}-47 \tau_{A}^{2}}{225}
\end{aligned}
$$

Note that both are concave in $\tau_{B}$. Solving $\pi_{A}^{*}(e, e)=\pi_{A}^{*}(m, m)$ w.r.t. $\tau_{B}$, we obtain:

$$
\tau_{B}^{A}=\frac{58 \tau_{A}-11 k \pm 15 \sqrt{2}\left(k-\tau_{A}\right)}{47}
$$

while $\pi_{B}^{*}(e, e)=\pi_{B}^{*}(m, m)$ in correspondence of

$$
\tau_{B}^{B}=\frac{58 \tau_{A}+4 k \pm 15 \sqrt{2}\left(k-\tau_{A}\right)}{62}
$$

and it can be quickly ascertained that $\tau_{B-}^{A}<\tau_{B-}^{B}<\tau_{A}<\widehat{\tau}<\tau_{B+}^{A}<\tau_{B+}^{B}$. This yields our final result:

Corollary 4 The upstream stage is a prisoners' dilemma for all $\tau_{B} \in\left(\tau_{A}, \widehat{\tau}\right)$.

\section{Concluding remarks}

We have reformulated the strategic delegation model by Sen and Stamatopoulos (2015), considering delegation as a continuous variable. On this basis, we have shown that the delegation stage replicates the structure of a prisoners' dilemma, yielding a unique subgame perfect equilibrium in which

\footnotetext{
${ }^{3}$ In fact, the question addressed in Lambertini and Trombetta (2002) is whether firms can avoid delegation in a supergame over an infinite time horizon.
} 
both firms delegate control to managers, the latter being given a mixedmotive contract. In such equilibrium, the more efficient firm makes higher profits than the less efficient rival.

\section{References}

[1] Fershtman, C. and K.L. Judd (1987), "Equilibrium incentives in oligopoly", American Economic Review, 77, 927-40.

[2] Lambertini, L. and M. Trombetta (2002), "Delegation and Firms' Ability to Collude", Journal of Economic Behavior and Organization, 47, 359-73.

[3] Sen, D. and G. Stamatopoulos (2015), "When an inefficient competitor makes higher profit than its efficient rival", Operations Research Letters, 43, 148-50.

[4] Sklivas, S. (1987), "The strategic choice of managerial incentives", Rand Journal of Economics, 18, 452-58.

[5] Vickers, J. (1985), "Delegation and the theory of the firm", Economic Journal, 95 (Conference Papers), 138-47. 


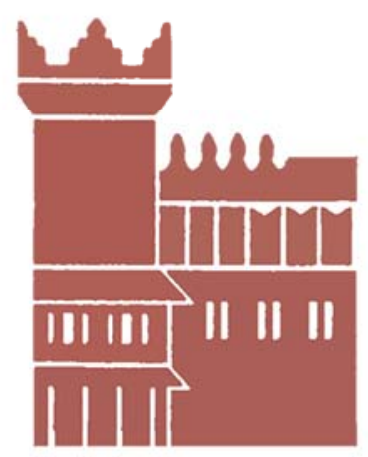

Alma Mater Studiorum - Università di Bologna DEPARTMENT OF ECONOMICS

Strada Maggiore 45

40125 Bologna - Italy

Tel. +39051 2092604

Fax +390512092664

http://www.dse.unibo.it 